\title{
ANÁLISIS DE SENSIBILIDAD DE LA ECUACIÓN ADITIVA Y MULTIPLICATIVA, UTILIZADAS PARA COMPROBAR EL ÍNDICE DE CALIDAD DE AGUA: CASO DEL HIDROTOPO DEL RÍO MENSABÉ
}

\author{
ANALYSIS OF SENSITIVITY OF THE ADDITIVE AND MULTIPLICATIVE EQUATION, \\ USED TO CHECK THE WATER QUALITY INDEX: CASE OF THE HYDROTOPE OF THE \\ MENSABÉ RIVER
}

\author{
Jaime A. Rivera Solís \\ Universidad de Panamá. Departamento de Geografía Física. CCIMBIO Centro de Capacitación, \\ Investigación y Monitoreo de la Biodiversidad, CRUV-UP. Panamá \\ jaime.rivera@up.ac.pa \\ https://orcid.org/0000-0002-8265-7836
}

\begin{abstract}
RESUMEN
Con trazado geoecológico, la pesquisa tuvo por objeto realizar un análisis integral de la calidad del agua de un tramo del río Mensabé, ubicado en su cuenca baja. Para tal fin, se determinaron sus características morfoclimáticas, y se efectuó el análisis de sensibilidad de las ecuaciones de Brown y Landwehr; recomendadas para obtener el índice de calidad del agua (ICA), atendiendo los parámetros de la National Sanitation Foundation (NSF). Se compilan los datos meteorológicos, y en ambiente ArcGIS 10.7.1., con el auxilio de la imagen SRTM (Shuttle Radar Topography Mission), y la imagen RapidEye, se delimita la cuenca, se localiza el hidrotopo y se deslinda la clasificación morfoclimática. Se utilizan dos puntos de muestreo y se colectan los nueve parámetros de la NSF para comprobar el ICA. Se calculan los resultados por medio de los dos métodos de agregación seleccionados (aditivo y multiplicativo), y, se emplea la ecuación de diferencia porcentual para estimar a través de éstos, el grado de sensibilidad de las fórmulas de agregación. Se comprueba que, el área de estudio pertenece a la región tropical húmeda seca, por estacionalidad; y que, la agregación multiplicativa demuestra mayor sensibilidad y restringe sus resultados en $\pm 17,28 \%$, en comparación con el método aditivo. Finalmente, al remplazar el parámetro de coliformes fecales por totales, se observa que la ecuación multiplicativa se torna más sensible, y ciñe los resultados en un $\pm 40,75 \%$.
\end{abstract}

PALABRAS CLAVE: Índice de calidad del agua, gestión integral del agua, cuenca hidrográfica, hidrotopo.

\begin{abstract}
With geoecological tracing, the research attempts to conduct a comprehensive analysis of the water quality of a stretch of the Mensabé River, located in its lower basin. To this end, its morphoclimatic characteristics were determined, and the sensitivity analysis of the equations of Brown and Landwehr; was performed, as recommended for obtaining the Water Quality Index (WQI), taking into account the parameters of the National Sanitation Foundation (NSF). The meteorological data is compiled, in an ArcGIS 10.7.1 environment, with the help of the SRTM (Shuttle Radar Topography Mission) image, and the RapidEye image, the basin is delimited, the hydrotrope is located and the morphoclimatic classification is demarcated. Two sampling points are used and the nine NSF parameters are collected to check the WQI. The results are calculated using the two aggregation methods (additive and multiplicative), selected, and, through these the percentage difference equation is used to estimate the degree of sensitivity of aggregation formulas. It is found that the study area belongs to the dry humid tropical region, seasonally; and that the multiplicative aggregation demonstrates greater sensitivity and restricts its results by $17.28 \%$, compared to the additive method. Finally, when replacing the fecal coliform parameter with totals, it is observed that the multiplicative equation becomes more sensitive, and closes the results by $40.75 \%$.
\end{abstract}


Rev. Col. Ciencia. Vol. 2, no. 1. Octubre 2020 - marzo 2021. ISSN L 2710-7434. pp. 26-42

KEYWORDS: Water quality index, comprehensive water management, watershed, hydrotope.

Artículo recibido: 26 de agosto de 2020.

Artículo aceptado: 10 de septiembre de 2020.

\section{INTRODUCCIÓN}

Las cuencas hidrográficas, son unidades espaciales que se caracterizan por la capacidad de captar, almacenar y distribuir el agua. Este recurso natural, se puede encontrar confinado dentro del subsuelo (dando origen a las aguas subterráneas), o expuesto sobre la superficie limitando los biomas lóticos (corrientes fluviales y pluviales), y biomas lenticos (lagos).

Para abordar el estudio de la cuenca hidrográfica como espacio natural, la Geografía Física, fundamenta sus procedimientos en el análisis del paisaje, entendido éste, como la unidad espacial donde interactúan los elementos bióticos y abióticos dentro del geosistema. El mismo, es un sistema espacial natural abierto, que se caracteriza por su estructura espacial (geomorfología), por su dinámica (asociada a la incidencia y flujo de energías: gravedad, radiación, vientos y agua...), y por la interdependencia entre sus elementos, incluyendo al hombre (Troppmair, 2012, p. 124). Esta concepción sistémica permite estudiar la realidad del paisaje por unidades espaciales las cuales se encuentran relacionadas entre sí (Mateo, Da Silva y Brito, 2010, p. 41), Al respecto, Christofoletti (1981), citado por Troppmair (2012), indica que "el estudio de los geosistemas es el objetivo fundamental de la Geografía Física" (p. 124).

A partir del geosistema, el análisis fisiográfico utiliza la cuenca hidrográfica como modelo de estudio (Molina, 1986, p. 76), o unidad de análisis espacial donde se aplica la escala témporoespacial para sustentar su topología y determinar los límites disociativos de todos sus componentes (Rivera, 2016, p. 144). Aunque, la escala témporo-espacial de Cailleux y Tricart, se confecciona con una marcada tendencia geomorfológica, deja observar que, el estudio local de las unidades pondera el geotopo (categoría G. VII - VIII), como la unidad espacial elemental, que corresponde al menor conjunto homogéneo del medio físico-químico (Bertrand y Bertrand, 2007, p. 15); y, el cual, para Mateo, et al (2010, p. 84), contiene en la composición abiótica del espacio, el estudio y diagnóstico ambiental del hidrotopo. Por consiguiente, para el manejo integral de la cuenca, a escala local, el hidrotopo constituye el espacio natural puntual de análisis. Al respecto, la Autoridad Nacional del Ambiente (2002), define la cuenca hidrográfica como...

Un área con características físicas, biológicas y geográficas debidamente delimitadas, donde interactúa el ser humano, en la cual las agua superficiales y subterráneas fluyen a una red natural mediante uno a varios causes de caudal continuo o intermitente, que confluyen a su vez a un curso mayor que desemboca en un río principal, en un deposito natural o artificial de agua, en un pantano o directamente en el mar. (Ley 44, artículo 2, numeral 1).

Las cuencas hidrográficas realizan diversas funciones, dentro de éstas, la producción de agua es primordial; puesto que, se considera el agua su recurso más valioso como líquido vital para sostener la vida en la tierra. Consecuentemente, a partir del reconocimiento de la cuenca hidrográfica como unidad de análisis espacial, el estudio geoecológico contempla el análisis de la calidad del agua, a través de su caracterización fisicoquímica y microbiológica, como un 
procedimiento metodológico indispensable para diagnosticar el estado ambiental del hidrotopo; y, en efecto, recomendar medidas en aras de garantizar el equilibrio ecológico del sistema.

Así, tanto el agua destinada para consumo humano dentro de la cuenca, como para uso recreativo incluyendo los ambientes marino costeros, tendrían un impacto negativo sobre la salud de los usuarios, si no se emplean métodos adecuados para el monitorear su calidad. La Organización Mundial de la Salud (OMS), contempla en las guías para ambientes seguros en agua recreativas el tema de la calidad del agua (específicamente contaminación por aguas residuales), como uno de los peligros a los que están expuestos los seres humanos (ANAM, 2006, p 2); además, "las enfermedades relacionadas con la contaminación del agua de consumo tienen una gran repercusión en la salud y las personas, por tanto, las medidas destinadas a mejorar la calidad del agua de consumo proporcionan beneficios significativos para la salud" (OMS, 2006, p. 11).

En Panamá, aunque se controlen todos los posibles focos de contaminación de las aguas corrientes; por génesis natural, debido a los altos índices de precipitación total anual (que como mínimo registran valores $>1200 \mathrm{~mm}$ ), que reciben las cuencas hidrográficas...

La calidad del agua puede variar con gran rapidez y todos los sistemas pueden presentar fallos ocasionales. Por ejemplo, la lluvia puede hacer aumentar en gran medida la contaminación microbiana en aguas de origen, y son frecuentes los brotes de enfermedades transmitidas por el agua después de periodos de lluvias. (OMS, 2006, p. 33).

Es a partir de dicha concepción, es cuando da inicio la diatriba sobre la toma de decisiones con respecto a cuál método se utilizará para evaluar la calidad del agua (físicoquímica y microbiológica, o por calidad biológica), a fin de garantizar su calidad ambiental desde el monitoreo del hidrotopo; independientemente del uso que se le dará al agua contenida en el sistema hídrico. Cabe resaltar que, los dos métodos citados son utilizados a nivel mundial, y, más que tener detractores, diversos autores coinciden que se complementan mutuamente. Por ejemplo, para Cornejo, et al., (2017), en Panamá...

No se puede evaluar la salud de las 52 cuencas hidrográficas del país a partir únicamente de las mediciones de parámetros fisicoquímicos y microbiológicos convencionales. Es importante el desarrollo de métodos que integren la estimación de la diversidad y densidad de diferentes grupos de organismos que puedan servir como indicadores, la evaluación del medio físico o calidad de hábitats, que definen las comunidades acuáticas presentes y la evaluación de agentes químicos tóxicos, entre otros.

Uno de los grupos más empleados como indicadores son los macro invertebrados dulceacuícolas, que son todos aquellos organismos invertebrados que pueden ser vistos a simple vista sin necesidad de un microscopio o una lente de aumento. Usualmente, miden por arriba de los $3 \mathrm{~mm}$ y llevan a cabo una parte o la totalidad de su ciclo de vida en el agua. (p. 13).

Sin embargo, esta investigación, no contempla el diagnóstico de la calidad del agua por calidad biológica, pues es un campo intrínseco asociado a las ciencias biológicas.

No obstante, de los denominados ambientes dulceacuícolas, se aborda el tema de la calidad del agua para los ambientes lóticos, a partir de la metodología fisicoquímica y microbiológica. Para tal fin, existen diversas técnicas que se distinguen por aplicar diferentes índices (por expresión 
matemática), dentro de los cuales se destacan, en PNMA II (2006, p. 2), los índices de calidad de agua de Horton, Toxidez, Prati, Dinius, Smith; y el de la National Sanitation Foundation (NSF), precisamente esta última, también referida por Sierra (2011, p. 161). No cabe duda que cada índice es valioso, pero, dentro de ellos, el índice de la NSF ha sido el más utilizado internacionalmente; Panamá, se suma a la lista de países que utilizan dicho índice para determinar la calidad del agua de sus corrientes fluviales, según la Autoridad Nacional del Ambiente (2005, p. 201) y Cornejo, et al. (2017, p. 20).

Desde el 2002 el Ministerio de Ambiente a través del laboratorio de calidad ambiental, monitorea las principales cuencas hidrográficas del país. Para el cálculo del índice de calidad de agua (ICA), se basan en la medición de parámetros fisicoquímicos y microbiológicos. (Cornejo, et al., 2017, p. 13). Así, en aras de garantizar la calidad del agua (para consumo humano), de las fuentes fluviales, la investigación discute una situación teórica - práctica (que aplica en la Rep. De Panamá), para evitar se generen conflictos de intereses entre los actores (Estado versus promotores privados), a la hora de escoger las variables (Subi), con sus respectivos pesos (Wi), y el tipo de ecuación, para determinar el índice de calidad de agua de un hidrotopo.

El problema en cuestión resulta puesto que, para obtener el índice propuesto inicialmente por la NSF, se aplica como técnica la ecuación aditiva de Brown, pero posteriormente, se propone utilizar también la ecuación multiplicativa de Landwehr. Por tanto, el trazado geoecológico de la pesquisa se fundamenta en el análisis integral de la calidad del agua de la cuenca baja del río Mensabé, el cual conlleva, puntualizar sus características morfoclimática, realizar el estudio comparativo de dos muestras de agua para determinar los ICA (por medio de las citadas ecuaciones), y fijar los costos por descontaminación. Así, se procura plantear las variables para documentar el análisis de sensibilidad, entendido como el "procedimiento sistemático para calcular los efectos que tendrán los métodos y datos elegidos en los resultados de un estudio" (MICI, 2003, p. 2), para alcanzar su objetivo.

\section{MATERIALES Y MÉTODOS}

\section{- $\quad$ Localización del área de estudio:}

En Geografía Física el análisis de la calidad del agua parte del tratado geosistémico del espacio natural, donde, a partir de la localización del hidrotopo, se realiza el análisis integral de la cuenca hidrográfica (según recorte espacial), procurando determinar las características fisiográficas del paisaje y correlacionar posibles causas de contaminación del agua. Al respecto, para Sierra (2011, p. 150), la evaluación de la calidad del agua contempla el análisis de los aspectos no acuáticos, donde se incluyen las características geomorfológicas y climáticas de la cuenca, la cobertura vegetal y el uso de suelos; comprendidos bajo el concepto de: Gestión integral de aguas.

El mapa de las cuencas hidrográficas de la República de Panamá cuenta con 58 polígonos, de los cuales 52 se encuentran codificados en atención al "Proyecto Hidrometeorológico Centroamericano (1967 - 1972), que unificó criterios para la numeración de las cuencas a nivel centroamericano" (ATLAS, 2016, p. 49). En la provincia de Los Santos, se ubica la cuenca hidrográfica . $^{\circ} 126$ (constituida entre los ríos La Villa y Tonosí), y dentro de ella, se localiza la subcuenca del río Mensabé; aguas las cuales, fueron el objeto estudio (Figura 1). 


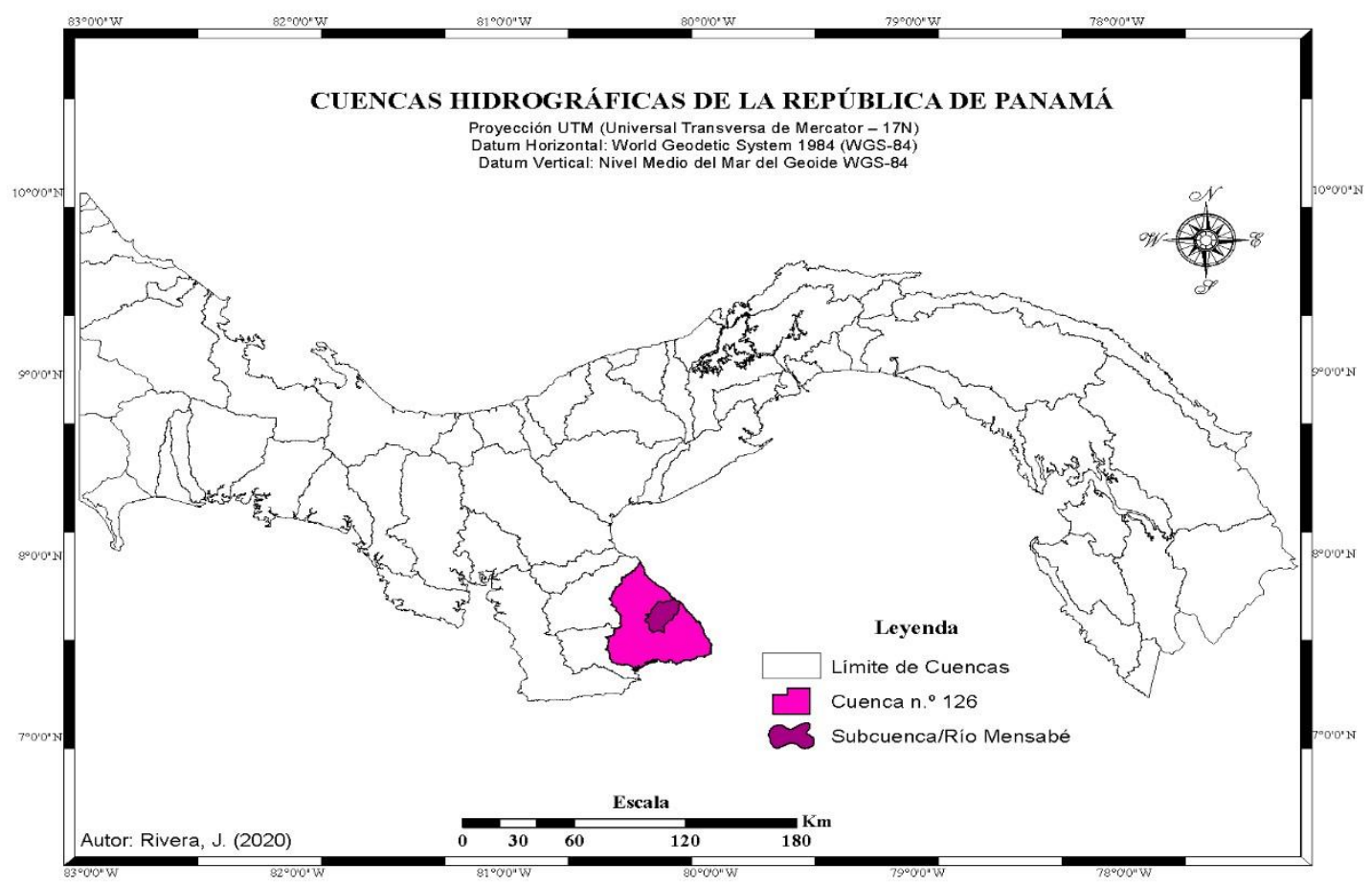

Figura 1. Localización del área de pesquisa. Autor: Rivera, J. (2020).

\section{- Materiales}

Para delimitar de la cuenca baja y localizar el hidrotopo: Raster/Imagen SRTM 1 Arc-Second Global (Shuttle Radar Topography Mission), resolución 30 m-recorte UTM - Zona 17 (USGS, 2012); Raster/Imagen RapidEye (2012), resolución de 5 m-recorte UTM - Zona 17; Software ArcGIS 10.7.1; Sistema de posicionamiento global, Garmin Etrex 20. GPS + GLONASS + WAAS; Cámara digital fotográfica HD. Canon SX30-IS; y, datos acumulados de precipitación total (Estación Pocrî), y temperatura promedio (Estación Pedasî).

Para determinar el ICA. Parámetros físicos: Turbidez (Turb./ FAU), y temperatura $\left(\mathrm{T} . /{ }^{\circ} \mathrm{C}\right), \mathrm{y}$ sólidos totales disueltos (S.T.D./ mg/L). Parámetros químicos: Potencial de hidrógeno (ph), nitratos (NO3/ mg/L), fosfatos (PO4/ mg/L), oxígeno disuelto (O.D./ \%), y demanda bioquímica de oxígeno (DBO5/ mg/L). Parámetro microbiológico y/o bacteriológico: Coliformes fecales (C.F./ UFC/100 mL), y coliformes totales (C.T./ NMP/100 mL).

Para fijar los costos por descontaminación: Sólidos suspendidos (S.S./ mg/L), demanda química de oxígeno (D.Q.O./ mg/L), y temperatura $\left(\mathrm{T} . /{ }^{\circ} \mathrm{C}\right)$.

\section{- Procedimientos metodológicos}

Sobre la cuenca:

El agua de las corrientes fluviales no necesariamente se encuentra en estado pura, siempre contiene sustancias contaminantes que provienen de diversas fuentes, vinculadas generalmente con el efecto erosivo del agua de lluvia y los vientos (Aguilo, et al., 1991, p. 280), asociados con la pendiente de la cuenca y su cobertura vegetal. Así, cuando se realiza el estudio de la calidad del 
agua del hidrotopo, también se incluye el análisis geoecológico de la cuenca; en aras de comprender el estado de los aspectos no acuáticos, vinculados con la calidad del agua.

De esta forma, se aplican técnicas de geoprocesamiento sobre la imagen SRTM 1 Arc-Second Global, para producir la red de triángulos a interpolar en ambiente ArcGIS 10.7.1. De allí, se extraen las curvas de nivel para obtener los datos hipsométricos, identificar vertientes y exponer la declividad. Las bandas espectrales de la imagen RapidEye (2012), se combinan en falso color combinadas B5/azul, B2/Rojo, y B4/verde, para discriminar la textura vegetal de la planicie fluviomarina sobre las planicies fluviales (Rivera, 2016, p. 34). Se utilizan los criterios morfométricos del Instituto de Investigaciones Tecnológicas (Brasil), para determinar la curva de nivel utilizada para recortar la cuenca y clasificar el tipo de relieve, según Tamiozzo, Marques y De Oliveira (2013).

Sobre el ICA:

En 1970, Brown, McClelland, Deininger e Tozer, presentan un índice de calidad (WQI, Water Quality Index); similar al de Horton, y financiado por la National Sanitation Foundation (NSF), (PNMA II, 2006, p. 23). Elaborado a partir de la evaluación de los 9 parámetros físicoquímicos y microbiológicos señalados (excepto, los coliformes totales), se convoca 142 expertos que utilizando la metodología Delphi, diseñan las curvas de calidad y especifican sus pesos correspondientes; resultados los cuales, se calculan inicialmente, a través de una ecuación de suma ponderada (Aguilo, et al., 1991, p. 294; Samboni, Carvajal y Escobar, 2007, p. 173).

Sin embargo, aunque en Panamá se aplique el ICA-NSF, al investigar el índice de calidad de agua, tanto promotores como fiscalizadores ambientales, deben consensuar, según Samboni, Carvajal y Escobar (2007, p. 172) "la selección de las variables, la determinación de los subíndices para cada parámetro y la elección de la fórmula de agregación”; la cual según SNET (2012, p. 4), la agregación de la información se realiza aplicando tanto la ecuación aditiva, como multiplicativa.

Por tanto, utilizando las recomendaciones del ICA-NSF, se trabaja con los parámetros seleccionados y la asignación de los pesos (Wi), los cuales corresponde al grado de importancia de los factores contaminantes del agua.

De esta forma, para calcular el ICA-NSF, se puede utilizar un promedio aritmético ponderado (Fórmula 1. Propuesta por Brown, et al., 1970), o el promedio multiplicativo ponderado (Fórmula 2), según Torres (2009, pp.9-10), y SNET (2012, p.5), propuesta por Landwehr y Deininger en 1976, con la cual demuestran que, dicha ecuación es mucho más sensible a los resultados de las variables estudiadas, y refleja con mayor precisión la calidad del agua.

- Fórmula 1... I.C.A. $=\sum_{i=1}^{9}\left(S u b_{i} * w_{i}\right)$

- Fórmula 2... I.C.A. $=\prod^{9}{ }_{i=1}\left(S u b_{i}{ }^{w_{i}}\right)$

Para determinar el valor del subíndice del parámetro, las respuestas del panel de expertos fueron promediadas en una escala de 0 - 100 (Subi), y se construyen las curvas de variación de calidad del agua para cada uno de los 9 parámetros (Figura 2).

Como queda expuesto en la tabla 1, los coliformes totales no son considerados dentro de los parámetros de calidad de agua de la NSF, aunque, según Díaz (2011, p.194), la NSF, también 
Rev. Col. Ciencia. Vol. 2, no. 1. Octubre 2020 - marzo 2021. ISSN L 2710-7434. pp. 26-42
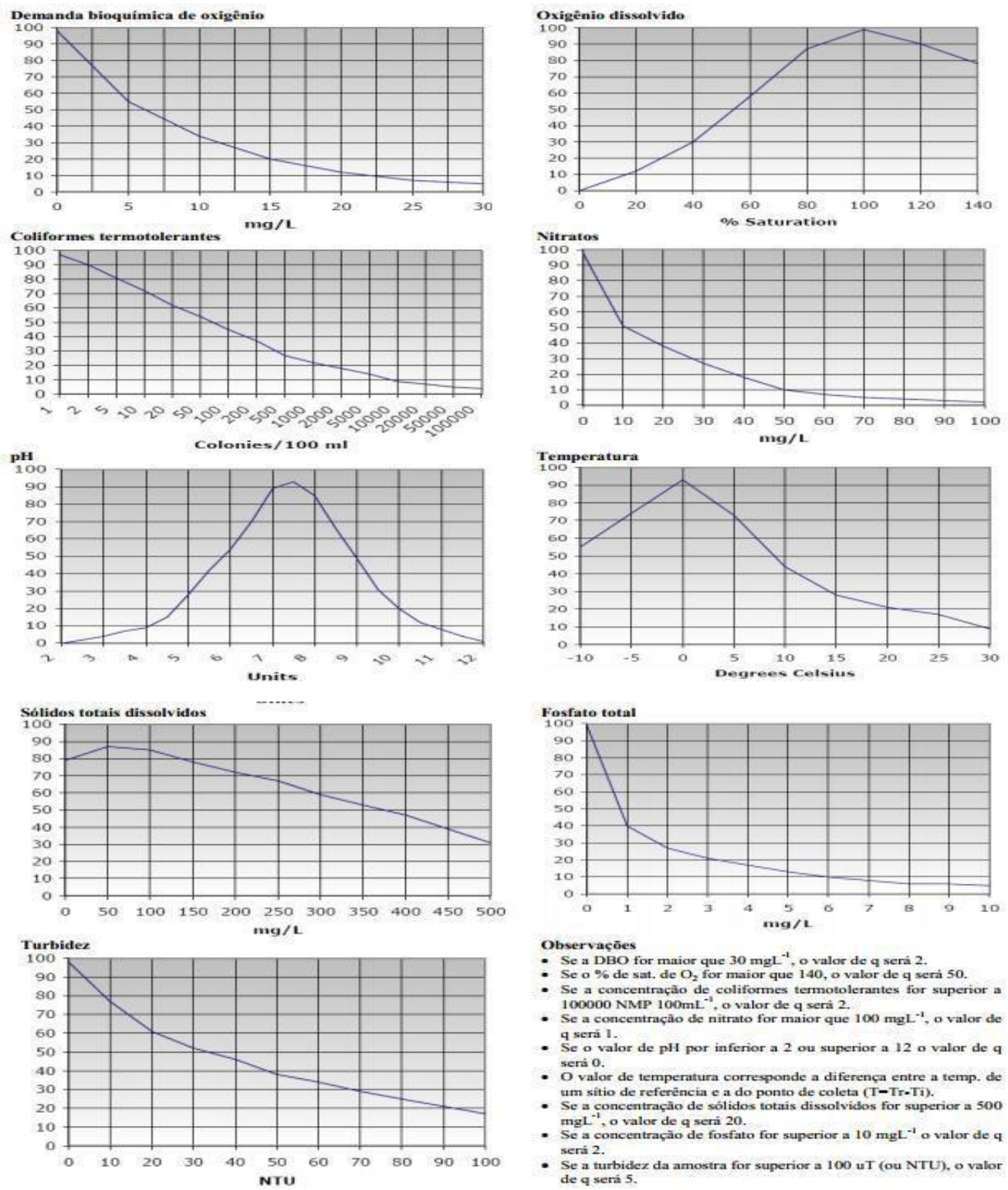

Observaçães

: Se a DBO for maior que $30 \mathrm{mgL}^{-1}$, o valor de $\mathrm{q}$ será 2 .
Se $\mathrm{O} \%$ de sat. de $\mathrm{O}$, for maior que 140 , o valor de $\mathrm{q}$ será 50

- Se a concentraçấ de coliformes termotolerantes for superior a

(c)

q será 1 .

- O valor de temperatura corresponde a diferença entre a temp. de

um sítio de referência e a do ponto de coleta ( $\mathrm{T}-\mathrm{Tr}$ - Ti).

ming , ovalor de q sed 20.

fosfato for superior a $10 \mathrm{mgL}^{-1}$ o valor de $\mathrm{q}$

Sen thent

de q seri 5 .

Figura 2: Curvas medias de variación de calidad del agua, NSF (2007). Fuente: Weinberg, A. (2013, p. 25).

considero dentro de los parámetros para definir el ICA, incluir los coliformes totales. Sin embargo, aunque los coliformes termotolerantes (coliformes fecales), son muy importantes en la evaluación de la calidad del agua como indicadores de contaminación fecal, sólo comprenden una porción del grupo de coliformes (Weinberg, 2013, p. 20). Ya asociados, son el recuento de los coliformes totales y fecales los mejores indicadores de contaminación fecal (Sierra, 2011, pp. 35 y 154). Al respecto, en Latinoamérica, ya se ha empleado la metodología para determinar el ICA, utilizando los coliformes totales. Se destacan los trabajos de ICA de Dinius, Montoya, y León. Cabe agregar que, Dinius y León, utilizan la ecuación del promedio multiplicativo ponderado como fórmula matemática para determinarlo (Samboni, Carvajal y Escobar, 2007, p. 177). 
Rev. Col. Ciencia. Vol. 2, no. 1. Octubre 2020 - marzo 2021. ISSN L 2710-7434. pp. 26-42

Tabla 1. Parámetros de ICA de la NSF y pesos asignados. Elaboración propia. Adaptado de (WRC, 2020).

\begin{tabular}{|c|c|}
\hline $\begin{array}{c}\text { Parámetros ICA- } \\
\text { NSF }\end{array}$ & $\begin{array}{c}\text { Peso } \\
\text { (Wi) }\end{array}$ \\
\hline $\begin{array}{c}\text { Oxígeno Disuelto (O.D./ } \\
\%)\end{array}$ & $\mathrm{W} 1=0,17$ \\
\hline $\begin{array}{c}\text { Coliformes Fecales } \\
\text { (C.F./ UFC/100 mL) } \\
\text { (NMP/100 mL) }\end{array}$ & $\mathrm{W} 2=0,16$ \\
\hline $\begin{array}{c}\text { Potencial de Hidrógeno } \\
(\mathrm{ph})\end{array}$ & $\mathrm{W} 3=0,11$ \\
\hline $\begin{array}{c}\text { Demanda Bioquímica de Oxígeno } \\
\text { (DBO5/ mg/L) }\end{array}$ & $\mathrm{W} 4=0,11$ \\
\hline $\begin{array}{c}\text { Cambio de Temperatura (T./ } \\
\left.{ }^{\circ} \mathrm{C}\right)\end{array}$ & $\mathrm{W} 5=0,10$ \\
\hline $\begin{array}{c}\text { Fosfatos (PO4/ } \\
\mathrm{mg} / \mathrm{L})\end{array}$ & $\mathrm{W} 6=0,10$ \\
\hline $\begin{array}{c}\text { Nitratos } \\
\text { (NO3/ mg/L) }\end{array}$ & $\mathrm{W} 7=0,10$ \\
\hline $\begin{array}{c}\text { Turbiedad (Turb./ } \\
\text { FAU) }\end{array}$ & $\mathrm{W} 8=0,08$ \\
\hline $\begin{array}{c}\text { Sólidos Totales Disueltos (S.T.D./ } \\
\mathrm{mg} / \mathrm{L})\end{array}$ & $\mathrm{W} 9=0,07$ \\
\hline
\end{tabular}

Por lo citado, primero, las nueve variables recomendadas por la NSF para obtener el ICA serán calculadas con la ecuación aditiva; éste índice será el resultado de referencia (grupo de control). Después, los parámetros citados serán calculados a través de la ecuación multiplicativa para determinar diferencias. Posteriormente, se utilizará como variable independiente el parámetro de coliformes totales en reemplazo de los coliformes fecales, manteniendo intacto los otros ocho parámetros y sus pesos. Los índices se obtendrán aplicando las dos ecuaciones y sus resultados interpretados a través la tabla comparativa de ICA de la NSF. (Figura 3).

Sobre costos por descontaminación:

Por la necesidad de establecer estimaciones económicas del valor de los recursos naturales que sufren impactos ambientales negativos, surgen diversos procedimientos y técnicas que los especialistas ambientales emplean para estimar los costos de reposición. Frecuentemente, por medio de la técnica Delphi, se consulta a los expertos, los cuáles deben determinar cuál será el método utilizado para establecer los costos por descontaminación del recurso afectado (Barzev, 2002, p.56).

Cuando además del ICA adquirido, se desea reflejar "la capacidad recuperadora de la corriente, el oxígeno disuelto o su porcentaje de saturación son indicadores muy apropiados para valorar esta 
actividad" (Sierra, 2011, p.153). Por tanto, debido a que, el ICA no necesariamente podría garantizar la existencia de las especies en in situ; para complementar el estudio de calidad de agua de la cuenca (o del tramo en estudio), es necesario evaluar (al margen de los resultados de las ecuaciones), los parámetros de sólidos suspendidos (S.S.), demanda química de oxígeno (D.Q.O.) y temperatura $\left({ }^{\circ} \mathrm{C}\right)$, como variables indispensables para calcular los costos de descontaminación del curso fluvial y garantizar la existencia de las especies.

\begin{tabular}{|c|c|}
\hline Criterio de uso & Rangos de Calidad \\
\hline Excelente & $90-100$ \\
\hline Buena & $70-89$ \\
\hline Media & $50-69$ \\
\hline Mala & $25-49$ \\
\hline Muy mala & $0-24$ \\
\hline
\end{tabular}

Figura 3: Rangos de calidad del agua para interpretar el ICA-NSF. Elaboración propia. Adaptado de (WRC, 2020).

El procedimiento se ejecuta, primero, determinando el tramo del curso fluvial que se desea descontaminar; a continuación, se georeferencian dos puntos de muestreo (aguas arriba y abajo del tramo), y se solicita la colecta de los parámetros SS, DQO y T ${ }^{\circ} \mathrm{C}$. Posteriormente, sí el análisis comparativo (entre los dos puntos), permite identificar un incremento en la cantidad de SS, se procede a calcular el volumen de agua, contaminada en el tramo, y se multiplica dicho volumen por el acrecentamiento de $\mathrm{SS}\left(\mathrm{Kg} / \mathrm{m}^{3}\right)$, entre los puntos. A seguir, el resultado permite inferir la cantidad aproximada de sólidos suspendidos $(\mathrm{Kg})$, vertidos en la corriente; los cuales a la postre, se multiplican por el costo (en dólares, y/o según moneda), promedio de descontaminación por kilogramo dispuesto por la autoridad competente. Finalmente, para complementar la evaluación del estado ambiental del hidrotopo, se utiliza el parámetro de temperatura $\left({ }^{\circ} \mathrm{C}\right)$, para obtener el OD (mg/L); y, se agrega el parámetro de DQO procurando establecer el importe de sustancias en suspensión que, son susceptibles químicamente a ser oxidadas.

\section{RESULTADOS}

A través de los procedimientos de geoprocesamiento sobre la imagen SRTM, se delimita la cuenca del río Mensabé, y se extraen las curvas de nivel, logrando obtener los parámetros de hipsometría, declividad, y realizar el recorte espacial de la cuenca baja empleando los criterios morfométricos (curva de nível $<20 \mathrm{msnm}$ ), recomendados por Tamiozzo, Marques y De Oliveira (2013, p. 300). A continuación, la máscara de corte creada sobre el polígono de la cuenca baja, se utiliza para recortar la imagen RapidEye; la cual, con la combinación de bandas recomendada en falso color, permite cuantificar la extensión de la planicie fluviomarina y la planicie fluvial. Además, se georeferencian los puntos de muestreo denominados: Muestra 2 (punto superior del curso), y muestra 1 (punto inferior del curso). (Figura 4). 


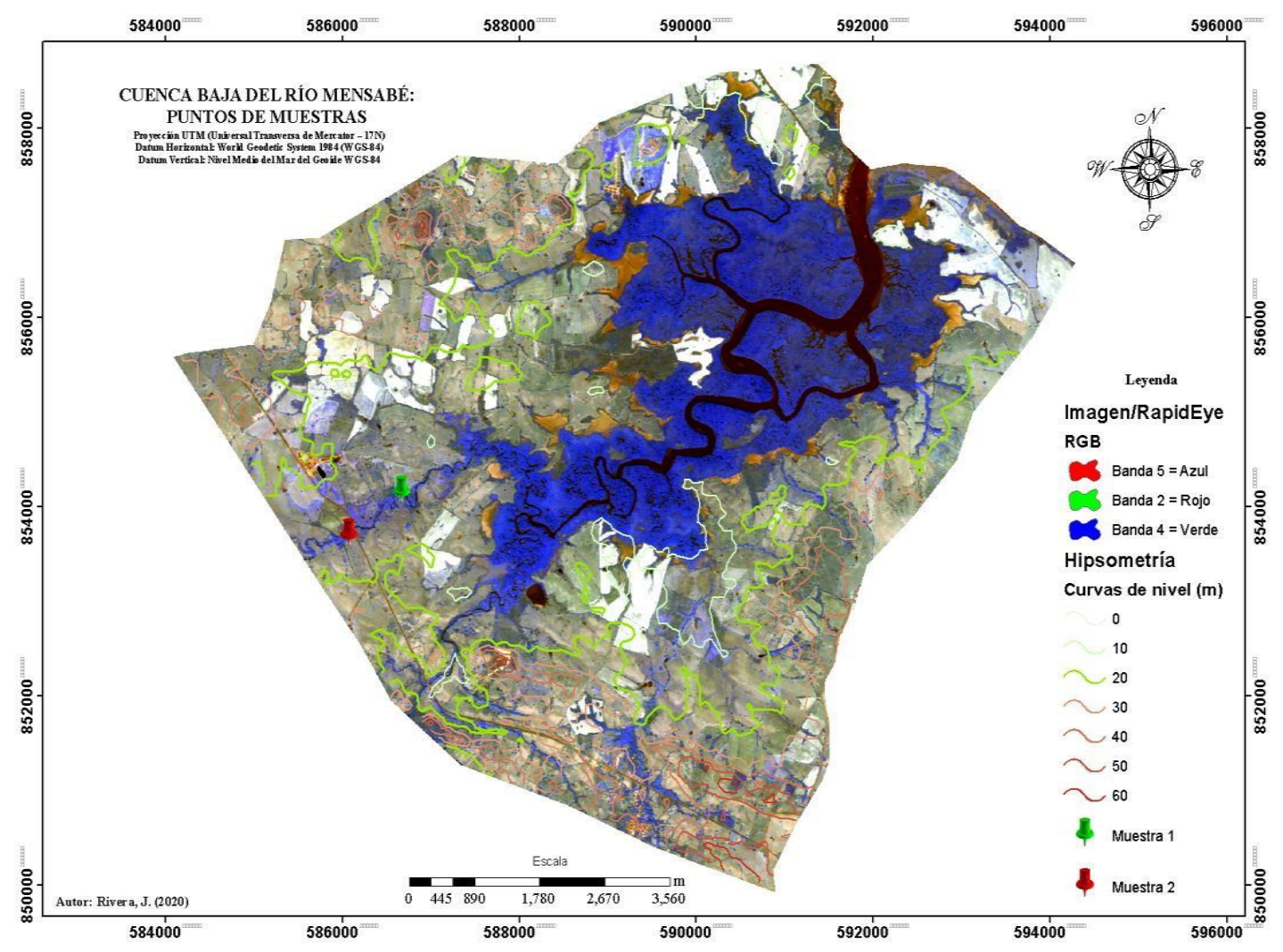

Figura 4: Cuenca baja del río Mensabé. Autor: Rivera, J. (2020).

Así, la cuenca baja (según recorte), del río Mensabé cuenta con 4993.01 ha., las cuales, se encuentran constituidas por una planicie fluvial de 2261.81 ha., y una planicie fluviomarina de 1092.32 ha.; dichas planicies en conjunto exponen valores de declividad o pendiente < 3\%. Por tanto, en atención al significado geomorfológico hipsométrico, las 1638.88 ha. restantes, detallan colinas suaves (curvas de nível > $20 \mathrm{msnm}$ ).

En atención al análisis de los datos meteorológicos acumulados (1970 - 2015), de precipitación total (estación Pocrí/ n. ${ }^{\circ}$ 126-002 / 70msnm), y temperatura promedio (estación Pedasí/ n. ${ }^{\circ} 126005$ / 47msnm); la cuenca baja del río Mensabé recibe aproximadamente $1293.3 \mathrm{~mm}$ de precipitación anual, con una temperatura media de $27.1^{\circ} \mathrm{c}$. Dichos datos climáticos (disponibles en http://www.hidromet.com.pa/), según Choley (1984), en Gutiérrez (2009, p. 444), caracterizan la región morfoclimática tropical húmeda seca (por estacionalidad). También, estas características climáticas evaluadas por medio del diagrama de los procesos morfológicos de Peltier (1950), en Gutiérrez (2009, p. 442), revelan que, en las regiones con precipitaciones >1000 mm la meteorización química tiene efectos de moderados, al igual que los movimientos de masas por erosión pluvial.

Se procede a despejar las fórmulas de agregación (Tablas 2,3,4 y 5), en las cuales, $\mathrm{W}_{\mathrm{i}}$, es una constante fija que representa el peso recomendado para cada parámetro; y, Subi, el subíndice del parámetro que se obtiene del cálculo del resultado de cada variable, por medio de los gráficos de curvas medias de variación de calidad del agua (figura 2). Seguidamente, el valor de Subi, es 
Rev. Col. Ciencia. Vol. 2, no. 1. Octubre 2020 - marzo 2021. ISSN L 2710-7434. pp. 26-42

multiplicado por $\mathrm{W}_{\mathrm{i}}$ (fórmula 1), y elevado a $\mathrm{W}_{\mathrm{i}}$ (fórmula 2), para obtener el valor del parámetro. Además, en las tablas 4 y 5, se remplaza el parámetro de coliformes fecales, por coliformes totales.

Respecto a los datos obtenidos para plantear los costos de recuperación ambiental del hidrotopo, tenemos que, la muestra 2 (Aguas arriba. UTM / N 853747 y E 586114), registra 20,0 (S.S./ mg/L), 16,8 (D.Q.O./ mg/L), y $27^{\circ} \mathrm{c}$ de temperatura; mientras que, la muestra 1 (Aguas abajo. UTM / N 854186 y E 586669), registra 16,0 (S.S./ mg/L), 16,2 (D.Q.O./ mg/L), y $25^{\circ} \mathrm{c}$ de temperatura. En consecuencia, se determina una disminución de la variable de sólidos suspendidos de $-4\left(\mathrm{Kg} / \mathrm{m}^{3}\right)$. En relación a la concentración de OD, con dependencia en la temperatura, Lind (1974), en Bain y Stevenson (1999, p. 186), la muestra 2 con $27^{\circ}$ c, contiene 7,86 O.D. (mg/L); y, la muestra 1 con $25^{\circ} \mathrm{c}$, concentran 8,11 O.D. (mg/L).

Tabla 2. Cálculo del ICA, según fórmula de agregación (muestra 1). Autor: Rivera, J. (2020).

\begin{tabular}{|c|c|c|c|c|c|}
\hline Parámetros ICA-NSF & $\mathrm{Wi}_{\mathrm{i}}$ & $\begin{array}{c}\text { Muestra } \\
1\end{array}$ & Subi & $\sum_{i=1}^{9}\left(S u b_{i} * w_{i}\right)$ & $\prod^{9}{ }_{i=1}\left(S u b_{i}^{w i}\right)$ \\
\hline $\begin{array}{c}\text { Oxígeno Disuelto (O.D./ } \\
\%)\end{array}$ & $\mathrm{W}_{1}=0,17$ & 55,4 & Sub1 $=53$ & 9,01 & 1,96 \\
\hline $\begin{array}{c}\text { Coliformes Fecales } \\
\text { (C.F./ UFC/100 mL) } \\
\text { (NMP/100 mL) }\end{array}$ & $\mathrm{W} 2=0,16$ & 7,0 & Sub2 $=77$ & 12,32 & 2 \\
\hline $\begin{array}{l}\text { Potencial de Hidrógeno } \\
\text { (ph) }\end{array}$ & $\mathrm{W} 3=0,11$ & 7,60 & Sub3 $=93$ & 10,23 & 1,64 \\
\hline $\begin{array}{c}\text { Demanda Bioquímica de Oxígeno } \\
\text { (DBO5/mg/L) }\end{array}$ & $\mathrm{W} 4=0,11$ & 8,8 & Sub4 $=41$ & 4,51 & 1,50 \\
\hline $\begin{array}{c}\text { Cambio de Temperatura } \\
\left(\text { T. } /{ }^{\circ} \mathrm{C}\right)\end{array}$ & $\mathrm{W} 5=0,10$ & 25,0 & Sub5 $=81$ & 8,1 & 1,55 \\
\hline $\begin{array}{c}\text { Fosfatos (PO4/ } \\
\mathrm{mg} / \mathrm{L})\end{array}$ & $\mathrm{W} 6=0,10$ & $<6,0$ & Sub6 $=10$ & 1 & 1,25 \\
\hline $\begin{array}{l}\text { Nitratos (NO3/ } \\
\mathrm{mg} / \mathrm{L})\end{array}$ & $\mathrm{W} 7=0,10$ & $<1,0$ & Sub7 $=99$ & 9,9 & 1,58 \\
\hline $\begin{array}{l}\text { Turbiedad (Turb./ } \\
\text { FAU) }\end{array}$ & $\mathrm{W} 8=0,08$ & 9,94 & Sub8 $=77$ & 6,16 & 1,41 \\
\hline $\begin{array}{c}\text { Sólidos Totales Disueltos (S.T.D./ } \\
\mathrm{mg} / \mathrm{L} \text { ) }\end{array}$ & $\mathrm{W}_{9}=0,07$ & 293,0 & Sub9 $=60$ & 4,2 & 1,33 \\
\hline & & & & $\mathrm{ICA}=65,43$ & $\mathrm{ICA}=55,35$ \\
\hline
\end{tabular}

Tabla 3. Cálculo del ICA, según fórmula de agregación (muestra 2). Autor: Rivera, J. (2020).

\begin{tabular}{|c|c|c|c|c|c|}
\hline $\begin{array}{c}\text { Parámetros ICA- } \\
\text { NSF }\end{array}$ & Wi & $\begin{array}{c}\text { Muestra } \\
2\end{array}$ & Subi & $\sum^{9_{i=1}\left(S u b_{i} * w_{i}\right)}$ & $\prod^{9_{i=1}\left(S u b_{i}{ }^{w i}\right)}$ \\
\hline $\begin{array}{c}\text { Oxígeno Disuelto } \\
\text { (O.D./ \%) }\end{array}$ & $\mathrm{W} 1=0,17$ & 56,0 & Sub1 $=54$ & 9,18 & 1,97 \\
\hline
\end{tabular}


Rev. Col. Ciencia. Vol. 2, no. 1. Octubre 2020 - marzo 2021. ISSN L 2710-7434. pp. 26-42

\begin{tabular}{|c|c|c|c|c|c|}
\hline $\begin{array}{c}\text { Coliformes Fecales } \\
\text { (C.F./ UFC/100 mL) } \\
(\mathrm{NMP} / 100 \mathrm{~mL})\end{array}$ & $\mathrm{W} 2=0,16$ & 5,0 & Sub2 $=80$ & 12,8 & 2,01 \\
\hline $\begin{array}{l}\text { Potencial de Hidrógeno } \\
\text { (ph) }\end{array}$ & $\mathrm{W} 3=0,11$ & 7,70 & Sub3 = 91 & 10,01 & 1,64 \\
\hline $\begin{array}{c}\text { Demanda Bioquímica de Oxígeno } \\
(\mathrm{DBO} 5 / \mathrm{mg} / \mathrm{L})\end{array}$ & $\mathrm{W} 4=0,11$ & 8,8 & Sub4 $=37$ & 4,07 & 1,48 \\
\hline $\begin{array}{c}\text { Cambio de Temperatura } \\
\left(\mathrm{T} . /{ }^{\circ} \mathrm{C}\right)\end{array}$ & $\mathrm{W} 5=0,10$ & 27,00 & Sub5 $=90$ & 9 & 1,56 \\
\hline $\begin{array}{c}\text { Fosfatos } \\
(\mathrm{PO} 4 / \mathrm{mg} / \mathrm{L})\end{array}$ & $\mathrm{W} 6=0,10$ & $<6,0$ & Sub6 $=10$ & 1 & 1,25 \\
\hline $\begin{array}{c}\text { Nitratos } \\
(\mathrm{NO} 3 / \mathrm{mg} / \mathrm{L})\end{array}$ & $\mathrm{W} 7=0,10$ & $<1,0$ & Sub7 $=99$ & 9,9 & 1,58 \\
\hline $\begin{array}{c}\text { Turbiedad (Turb./ } \\
\text { FAU) }\end{array}$ & $\mathrm{W} 8=0,08$ & 10,60 & Sub8 $=74$ & 5,92 & 1,41 \\
\hline $\begin{array}{c}\text { Sólidos Totales Disueltos } \\
\text { (S.T.D./ mg/L) }\end{array}$ & $\mathrm{W} 9=0,07$ & 208,0 & Sub9 $=72$ & 5,04 & 1,34 \\
\hline & & & & $\mathrm{ICA}=66,92$ & $\mathrm{ICA}=55,94$ \\
\hline
\end{tabular}

Tabla 4. Cálculo del ICA, según fórmula de agregación, utilizando coliformes totales (muestra 1). Autor: Rivera, J. (2020).

\begin{tabular}{|c|c|c|c|c|c|}
\hline $\begin{array}{c}\text { Parámetros ICA- } \\
\text { NSF }\end{array}$ & $\mathrm{Wi}_{\mathrm{i}}$ & $\begin{array}{c}\text { Muestra } \\
1\end{array}$ & Subi & $\sum^{g_{i=1}}\left(\operatorname{Su} b_{i} * w_{i}\right)$ & $\prod^{9}{ }_{i=1}\left(S u b_{i}{ }^{w i}\right)$ \\
\hline $\begin{array}{l}\text { Oxígeno Disuelto } \\
\text { (O.D. } / \%)\end{array}$ & $\mathrm{W}_{1}=0,17$ & 55,4 & Sub1 $=53$ & 9,01 & 1,96 \\
\hline $\begin{array}{l}\text { Coliformes Totales } \\
\text { (C.T./ NMP/100 mL) }\end{array}$ & $\mathrm{W} 2=0,16$ & 461110,00 & Sub2 $=3$ & 0,48 & 1,19 \\
\hline $\begin{array}{l}\text { Potencial de Hidrógeno } \\
(\mathrm{ph})\end{array}$ & $\mathrm{W} 3=0,11$ & 7,60 & Sub3 $=93$ & 10,23 & 1,64 \\
\hline $\begin{array}{c}\text { Demanda Bioquímica de Oxígeno } \\
(\mathrm{DBO} 5 / \mathrm{mg} / \mathrm{L})\end{array}$ & $\mathrm{W} 4=0,11$ & 8,8 & Sub4 $=41$ & 4,51 & 1,50 \\
\hline $\begin{array}{l}\text { Cambio de Temperatura } \\
\left(\mathrm{T} . /{ }^{\circ} \mathrm{C}\right)\end{array}$ & $\mathrm{W} 5=0,10$ & 25,0 & Sub5 $=81$ & 8,1 & 1,55 \\
\hline $\begin{array}{c}\text { Fosfatos } \\
(\mathrm{PO} 4 / \mathrm{mg} / \mathrm{L})\end{array}$ & $\mathrm{W} 6=0,10$ & $<6,0$ & Sub6 $=10$ & 1 & 1,25 \\
\hline $\begin{array}{c}\text { Nitratos } \\
(\mathrm{NO} 3 / \mathrm{mg} / \mathrm{L})\end{array}$ & $\mathrm{W} 7=0,10$ & $<1,0$ & Sub7 $=99$ & 9,9 & 1,58 \\
\hline $\begin{array}{c}\text { Turbiedad } \\
\text { (Turb./FAU) }\end{array}$ & $\mathrm{W} 8=0,08$ & 9,94 & Sub8 $=77$ & 6,16 & 1,41 \\
\hline $\begin{array}{l}\text { Sólidos Totales Disueltos } \\
\text { (S.T.D./ } \mathrm{mg} / \mathrm{L} \text { ) }\end{array}$ & $\mathrm{W}_{9}=0,07$ & 293,0 & Sub9 $=60$ & 4,2 & 1,33 \\
\hline & & & & ICA $=53,59$ & $\mathrm{ICA}=32,93$ \\
\hline
\end{tabular}


Rev. Col. Ciencia. Vol. 2, no. 1. Octubre 2020 - marzo 2021. ISSN L 2710-7434. pp. 26-42

Tabla 5. Cálculo del ICA, según fórmula de agregación, utilizando coliformes totales (muestra 2). Autor: Rivera, J. (2020).

\begin{tabular}{|c|c|c|c|c|c|}
\hline $\begin{array}{c}\text { Parámetros ICA- } \\
\text { NSF }\end{array}$ & $\mathrm{Wi}_{\mathrm{i}}$ & $\begin{array}{l}\text { Muestra } \\
2\end{array}$ & Subi & $\sum^{9_{i=1}}\left(S u b_{i} * w_{i}\right)$ & $\prod^{9}{ }_{i=1}\left(S u b_{i}{ }^{w i}\right)$ \\
\hline $\begin{array}{l}\text { Oxígeno Disuelto } \\
\text { (O.D./\%) }\end{array}$ & $\mathrm{W}_{1}=0.17$ & 56,0 & Sub1 $=54$ & 9,18 & 1,97 \\
\hline $\begin{array}{l}\text { Coliformes Totales } \\
\text { (C.T./ NMP/100 mL) }\end{array}$ & $\mathrm{W} 2=0.16$ & 15760,00 & Sub2 = 9 & 1,44 & 1,42 \\
\hline $\begin{array}{l}\text { Potencial de Hidrógeno } \\
\text { (ph) }\end{array}$ & $\mathrm{W} 3=0.11$ & 7,70 & Sub3 = 91 & 10,01 & 1,64 \\
\hline $\begin{array}{c}\text { Demanda Bioquímica de Oxígeno } \\
\text { (DBO5 } / \mathrm{mg} / \mathrm{L})\end{array}$ & $\mathrm{W} 4=0.11$ & 8,8 & Sub4 $=37$ & 4,07 & 1,48 \\
\hline $\begin{array}{l}\text { Cambio de Temperatura } \\
\left(\mathrm{T} . /{ }^{\circ} \mathrm{C}\right)\end{array}$ & $\mathrm{W} 5=0.10$ & 27,00 & Sub5 $=90$ & 9 & 1,56 \\
\hline $\begin{array}{c}\text { Fosfatos } \\
(\mathrm{PO} 4 / \mathrm{mg} / \mathrm{L})\end{array}$ & $\mathrm{W} 6=0.10$ & $<6,0$ & Sub6 $=10$ & 1 & 1,25 \\
\hline $\begin{array}{c}\text { Nitratos } \\
(\mathrm{NO} 3 / \mathrm{mg} / \mathrm{L})\end{array}$ & $\mathrm{W} 7=0.10$ & $<1,0$ & Sub7 $=99$ & 9,9 & 1,58 \\
\hline $\begin{array}{l}\text { Turbiedad } \\
\text { (Turb./FAU) }\end{array}$ & $\mathrm{W} 8=0.08$ & 10,60 & Sub8 $=74$ & 5,92 & 1,41 \\
\hline $\begin{array}{l}\text { Sólidos Totales Disueltos } \\
\text { (S.T.D./ mg/L) }\end{array}$ & $\mathrm{W} 9=0.07$ & 208,0 & Sub9 $=72$ & 5,04 & 1,34 \\
\hline & & & & $\mathrm{ICA}=55,56$ & $\mathrm{ICA}=39,52$ \\
\hline
\end{tabular}

\section{DISCUSIÓN}

El 67,18 \% de la cuenca baja del río Mensabé contiene los paisajes naturales de planicies, donde el $21,88 \%$ corresponde a la planicie fluviomarina, cubierta por mangle; y el 45,30 \% conciernen a la planicie fluvial, revestida de herbáceas, arbustos y árboles dispersos, los cuales exhiben densidad continua sólo en el bosque de galería. En estas planicies con valores de declividad < 3\%, el movimiento acelerado de la masa de suelo por la acción de la gravedad, no refleja cicatrices en el terreno; por el contrario, se acumulan las partículas sobre la planicie fluvial formando aluviones, y sobre la planicie fluviomarina suelos halomórficos. Pero, las laderas que afloran de las colinas suaves, que representan el 32,82 \% de la cuenca baja, con declividad entre 10 y $12 \%$, (con uso agropecuario), se consideran zonas de pendiente moderada; las cuales, con precipitación concentradas en 8 meses $(1293,3 \mathrm{~mm})$, desencadenan el movimiento del detrito y coladas de barro (aflorando la roca, y generando erosión por cárcavas), y posteriormente erosión difusa sobre la planicie fluvial. Al respecto, Fournier (1960), en Gutiérrez, (2009, p. 444), indica que los trópicos húmedos estacionales presentan los valores más elevados de erosión del suelo estimados de 100 500 ton/ $/ \mathrm{km}^{2} /$ año. Finalmente, en esta región morfoclimática, es el fenómeno de hidratación sobre el suelo y la roca, el principal responsable del proceso de meteorización, que provoca la reducción del material, y lo prepara para ser transportado y depositado sobre las planicies y la corriente fluvial. 
Al comparar los cálculos de ICA, expuestos en las tablas 2,3,4 y 5, con los rangos de calidad del agua (figura 3), es posible comprobar que, en los sitios de muestreo (1 y 2), utilizando coliformes fecales y ambos métodos de agregación (fórmulas 1 y 2), el ICA se encuentra en rango medio (entre 50 y 69). No obstante, al utilizar coliformes totales con la ecuación aditiva, el ICA aún se encuentra en rango medio; pero, al implementar la ecuación multiplicativa, el ICA disminuye su rango entre 25 y 49 , y es considera mala.

Con este antecedente, se proyecta realizar el análisis de sensibilidad entre las fórmulas de agregación, y la inclusión de los coliformes totales como variable independiente. Así, se evaluó la diferencia porcentual (fórmula 3), entre los ICA obtenidos por punto de muestreo, considerados como valores absolutos, que resultan al aplicar diferentes metodologías para obtener un resultado.

$$
\begin{aligned}
& \text { - Fórmula } 3 \ldots\left|\frac{x_{1}-x_{2}}{\left(\frac{x_{1}+x_{2}}{2}\right)}\right| \times 100=\% \text { de diferencia entre } x_{1} \text { y } x_{2} \\
& \text { - } \quad \text { Ejemplo ... }\left|\frac{65,43-55,35}{\left(\frac{65,43+55,35}{2}\right)}\right| \times 100=\%
\end{aligned}
$$

En consecuencia, al despejar la fórmula, con los resultados de la tabla 2, se genera una diferencia porcentual de $16,69 \%$; en la tabla 3 , de $17,87 \%$; en la tabla 4, de $47,76 \%$; y en la tabla 5 , de 33,74 \%. Por tanto, en los sitios de muestreo (1 y 2), utilizando los coliformes fecales, se demuestra que, al utilizar como método de agregación la ecuación multiplicativa, ésta, es más sensible y restringe los resultados en $\pm 17,28 \%$. Por otro lado, empleando los coliformes totales, en los citados sitios, observamos que, la ecuación multiplicativa, se torna aún más sensible, y ciñe los resultados en un $\pm 40,75 \%$.

Sin más, debido a que los cálculos para determinar los costos de recuperación ambiental de un hidrotopo, se deben fundamentar en un análisis comparativo para demostrar el incremento de S.S. y D.Q.O, versus, la disminución en la concentración de oxígeno disuelto O.D. (mg/L); tenemos que, como los resultados exponen un decrecimiento de los S.S. de - $4 \mathrm{Kg} / \mathrm{m}^{3}$ entre la muestra 2 (agua arriba), y la muestra 1, (aguas abajo, ubicada a una distancia 1046,28 m), no es posible generar o calcular costos por descontaminación, porque, de forma natural, tras un recorrido de \pm 1 $\mathrm{km}$ de longitud, las aguas del tramo se han autodepurado. De igual forma acontece con el D.Q.O, que decreció en $0,6 \mathrm{mg} / \mathrm{L}$, y la concentración de O.D, que aumenta $0.25 \mathrm{mg} / \mathrm{L}$.

\section{CONCLUSIONES}

A través de la caracterización morfoclimática de la cuenca, se revela que la zona pertenece a la región tropical húmeda seca. Sin embargo, se debe aclarar que se denomina seco por estacionalidad climática, es decir, que presentan de dos a cinco meses con precipitaciones inferiores a $50 \mathrm{~mm}$; justo durante el invierno del hemisferio norte (periodo mal denominado en Panamá como verano). 
Por tanto, esta designación de "seco", no debe interpretarse como una zona con bajos índices de precipitación total anual, puesto que, registra datos $>1000 \mathrm{~mm}$ anuales.

Los cursos fluviales de los trópicos húmedos secos, por naturalidad, poseen un sistema de autodepuración o tratamiento espontaneo, asociado a la estacionalidad climática. Pero, en atención a las características morfométricas de la cuenca, las copiosas lluvias concentradas en 8 meses y las actividades agropecuarias sin fiscalización técnica; estas, podrían considerarse las causas responsables por el aumento de los S.S., los coliformes totales, la D.Q.O., y la disminución del O.D., en el tramo fluvial evaluado. Al respecto, sobre, la concentración de los parámetros citados, empleados para evaluar el óptimo funcionamiento del bioma lótico, se debe puntualizar que, todos se encuentran por debajo de los valores máximos permisibles de efluentes líquidos, en MINSA (2000, p.p. 38 - 39), S.S./ mg/L < 35 y D.Q.O./ mg/L < 100. En el caso del O.D., los valores > 5 (mg/L), son considerados adecuados para el desarrollo de la vida de la mayoría de las especies de peces y organismos acuáticos.

Los coliformes fecales (en especial la E. coli), se relaciona directamente con la eliminación de heces sobre el agua, de otra forma, no sería frecuente encontrarlo. Sin embargo, los coliformes totales (aunque no necesariamente de procedencia fecal), se encuentran con mayor asiduidad sobre la totalidad de la cuenca; por lo que, su cuantificación e inclusión como parámetro para determinar el ICA, podría garantizar un agua de mejor calidad para el abastecimiento público. Cabe agregar que, los criterios establecidos en la figura 3, son considerados generales, y, por tanto, existen diversas teorías que proponen interpretar los criterios en atención al uso directo que se le dará al agua de la cuenca. Por ejemplo, este estudio de caso, planteo el uso como criterio para consumo humano; por tanto, para el abastecimiento de la red, se recomienda que el ICA sea > 50.

Finalmente, impera la necesidad de reglamentar entre los actores y autoridades responsables, los parámetros, sus pesos y el método de agregación para definir el índice de calidad de agua (ICA), según destino final; toda vez que, el análisis de sensibilidad entre las fórmulas de agregación, y la inclusión de los coliformes totales, demostraron una diferencia porcentual que oscila entre el 17,28 $\%$, y el 40,75\%.

\section{REFERENCIAS}

Aguilo, M.; Aramburu, M.; Ayuso, E.; Blanco, A.; Calatayud, T.; Ceñal, M., ... Sáiz, M. (1991). Guía para la Elaboración de Estudios del Medio Físico: Contenido y Metodología. 3 ed. Madrid: Editora Secretaría General Técnica, Centro de Publicaciones, Ministerio de Obras Públicas y Transporte.

Bain, M., y Stevenson, N. (1999). Aquatic habitat assessment: Common methods. Maryland, United States of America. American Fisheries Society.

Barzev, R. (2002). Guía metodológica de valoración económica de bienes, servicios e impactos ambientales. Managua, Nicaragua: Corredor biológico mesoamericano. Oficina Regional de Coordinación.

Brasil (2006). Governo do Estado de Pernambuco. Programa Nacional do Meio Ambiente (PNMA II). Índice e Indicadores de Qualidade de Água: Revisão da Literatura. Recuperado de: 
Rev. Col. Ciencia. Vol. 2, no. 1. Octubre 2020 - marzo 2021. ISSN L 2710-7434. pp. 26-42

http://www.cprh.pe.gov.br/downloads/indice-agua-volume1.pdf

Bertrand, G. y Bertrand, C. (2007). Uma Geografia Transversal e de Travessias: o meio ambiente através dos territórios e das temporalidades. Brasil: Editora Massoni.

Cornejo, A.; López, E.; Ruiz, R.; Sedeño, J.; Armitage, B.; Arefina, T., ... Avila, I. (2017). Diagnóstico de la condición ambiental de los afluentes superficiales de Panamá. Panamá: Instituto Conmemorativo Gorgas de Estudios de la Salud y Ministerio de Ambiente. Recuperado de: https://fliphtml5.com/eebm/obrj/basic

Díaz, R. (2011). Desarrollo Sustentable. Una Oportunidad para la Vida. 2 ed. Mexico D.F.: McGraw-Hill.

El Salvador. (2012). Servicio Nacional de Estudios Territoriales (SNET). Ministerio de Medio Ambiente y Recursos Naturales. Índice de Calidad del Agua General "ICA”.

Recuperado de: http://www.snet.gob.sv/Hidrologia/Documentos/calculoICA.pdf

Gutiérrez, M. (2009). Geomorfología. Madrid, España: Pearson Educación.

Instituto Geográfico Nacional "Tommy Guardia". (2016). Atlas Nacional de la República de Panamá. (5 $5^{\mathrm{a}}$ ed). Colombia: Impresiones Carpal.

Mateo, J.; Da Silva, E. y Brito, A. (2010). Geoecologia das paisagens: Uma visão geossistêmica da análise ambiental. ( $3^{\mathrm{a}}$ ed.). Fortaleza, Brasil: Edições UFC.

Molina, M. (1986). Paisaje y región: Una aproximación conceptual y metodológica. En teoría y práctica de la Geografía (pp. 63 - 87). Madrid, España: Editorial Alhambra, S.A.

Organización Mundial de la Salud. (2006). Guías para la calidad del agua potable. (3 ${ }^{\mathrm{a}}$ ed.). Volumen $1 . \quad$ Recuperado de: https://www.who.int/water_sanitation_health/dwq/gdwq3_es_fulll_lowsres.pdf?ua=1

Panamá. Autoridad Nacional del Ambiente. (5 de agosto de 2002). (Ley 44 de 5 de agosto de 2002). Ley por medio de la cual se establece el régimen administrativo especial para el manejo, protección y conservación de las cuencas hidrográficas de la República de Panamá. [Ley 44 de 2002]. Recuperado de: http://consulweb.miambiente.gob.pa/imagenes/legal/484.pdf

Panamá. (2005). Autoridad Nacional del Ambiente. Laboratorio de calidad ambiental. II informe de monitoreo de la calidad del agua 2004 - 2005. República de Panamá, Panamá.

Panamá. (2006). Autoridad Nacional del Ambiente. Documento técnico científico: Elaboración del anteproyecto de norma de calidad ambiental y límites máximos permisibles de aguas continentales para uso recreativo con y sin contacto directo. República de Panamá, Panamá.

Panamá. Ministerio de Comercio e Industrias. Dirección General de Normas y Tecnología Industrial. (23 de junio de 2003). Resolución que adopta la norma técnica DGNTICOPANIT-ISO-14041-2003. Gestión medioambiental y análisis de ciclo de vida. [Resolución 303 de 2003]. 
Rev. Col. Ciencia. Vol. 2, no. 1. Octubre 2020 - marzo 2021. ISSN L 2710-7434. pp. 26-42

Panamá. (2000). Ministerio de Salud. Sub-Dirección General de Salud Ambiental. Normas para aguas residuales. Reglamento técnico DGNTI-COPANIT 35 - 2000.

Rivera, J. (2016). Avaliação geoecológica aplicada à ordenação ambiental da paisagem marinhocosteira: caso da bacia hidrográfica do Rio Purio Província dos Santos - Panamá (Tese pra o título de doutor em Geografía). Recuperado de: https://repositorio.unesp.br/handle/11449/143897

Samboni, N.; Carvajal, Y. y Escobar, J. (2007). Revisión de parámetros fisicoquímicos como indicadores de calidad y contaminación del agua. Revista Ingeniería e Investigación, 27

(3), 172-181. Recuperado de: https://repositorio.unal.edu.co/bitstream/handle/unal/28869/14858-447401PB.pdf?sequence $=1 \&$ is Allowed $=y$

Sierra, C. (2011). Calidad del agua: Evaluación y diagnóstico. Medellín, Colombia: Universidad de Medellín, ediciones de la U.

Tamiozzo, F.; Marques, R. y De Oliveira, S. (2013). Introdução à Geomorfologia. São Paulo: Cengage Learning.

Torres, F. (2009). Desarrollo y aplicación de un índice de calidad de agua para ríos en Puerto Rico. (Tese para el título de magister en Ciencia para Ingeniería Civil). Recuperado de: http://prwreri.uprm.edu/publications/PR 2009 01.pdf

Troppmair, H. (2012). Biogeografia e Meio ambiente. ( $9^{\text {a }}$ ed.). Rio de Janeiro, Brasil: Technical Books.

U.S.Geological Survey (USGS). (2012). Shuttle Radar Topography Mission (SRTM). United States. U.S. Departmente of the Interior. Disponível em: 〈https://lta.cr.usgs.gov〉.

Water Research Center (WRC). (2020). Calculating NSF Water Quality Index (WQI).

Recuperado de: https://www.water-research.net/watrqualindex/index.htm

Weinberg, Á. (2013). Uso de índices de qualidade de água para a caracterização da bacia hidrográfica do rio Guandu. (Tese pra o título de Engenharia Ambiental). Recuperado de: http://www.drhima.poli.ufrj.br/images/documentos/tcc/2013/agatha-weinberg-2013.pdf 